\title{
MicroRNA-21 regulation of the progression of viral myocarditis to dilated cardiomyopathy
}

\author{
HONG-FEI XU ${ }^{1}$, YU-JIE DING ${ }^{2 *}$, ZHI-XIANG ZHANG ${ }^{1}$, ZU-FENG WANG $^{1}$, CHENG-LIANG LUO ${ }^{3}$, \\ BEI-XU LI ${ }^{3}$, YI-WEN SHEN ${ }^{3}$, LU-YANG TAO ${ }^{1}$ and ZI-QIN ZHAO ${ }^{3 *}$ \\ ${ }^{1}$ Department of Forensic Medicine, Soochow University, Suzhou Dushuhu High Educational Town, Suzhou, Jiangsu 215123; \\ ${ }^{2}$ Department of Medical Cosmetology, Affiliated Hospital of Nantong University, Nantong, Jiangsu 226001; ${ }^{3}$ Department of \\ Forensic Medicine, Shanghai Medical College, Fudan University, Xuhui, Shanghai 200032, P.R. China
}

Received September 3, 2013; Accepted March 13, 2014

DOI: $10.3892 / \mathrm{mmr} .2014 .2205$

\begin{abstract}
MicroRNAs (miRNAs) comprise a broad class of small non-coding RNAs that control the expression of complementary target messenger RNAs. The dysregulation of miRNAs by several mechanisms has been described in various disease states, including cardiac disease. Although an etiological link between viral myocarditis (VMC) and idiopathic dilated cardiomyopathy (DCM) has long been recognized, the true extent of this association is uncertain. Previous studies of the two diseases have focused on protein degradation systems. In the present study, miR-21 expression and its potential role in VMC and DCM was investigated. The expression levels of miR-21, its target gene sprouty homolog 1 (SPRY1) and mitogen-activated protein kinase (MAPK) were measured by quantitative polymerase chain reaction. The protein levels of SPRY1 and MAPK were also determined by western blotting. miR-21 levels were significantly increased in cardiac myocytes from VMC and DCM in comparison with control samples. The levels of SPRY1 were decreased and MAPK activity was increased. Using a bioinformatics-based approach, an identical potential binding site was identified in mouse miR-21 and the SPRY 3' untranslated region (3' UTR), suggesting a regulatory role for miR-21. In cultured, miRNA-transfected myocardial cells, the overexpression of miR-21 was associated with a decrease in SPRY1 protein expression and an increased expression of the MAPK protein. These findings revealed that
\end{abstract}

Correspondence to: Professor Zi-Qin Zhao, Department of Forensic Medicine, Shanghai Medical College, Fudan University, 138 Yixueyuan Road, Xuhui, Shanghai 200032, P.R. China

E-mail:whhongfei@sina.com

Dr Yu-Jie Ding, Department of Medical Cosmetology, Affiliated Hospital of Nantong University, 20 Xisi Road, Nantong, Jiangsu 226001, P.R. China

E-mail:whatexhf@sina.com

*Contributed equally

Key words: microRNA, viral myocarditis, dilated cardiomyopathy, SPRY, miRNA-21 changes in the expression of miRNAs may contribute to the pathogenesis of VMC to DCM and establish the therapeutic efficacy of miRNA targeted intervention in a cardiovascular disease setting.

\section{Introduction}

Viral myocarditis (VMC), a disease without reliable or effective treatment, causes chronic dilated cardiomyopathy (DCM) or mortality in up to $20 \%$ of affected children and $50 \%$ of affected adults (1). The events producing cytopathic effects and leading to cardiac dysfunction following viral attachment and replication are not clearly understood. DCM is a disease of the heart muscle characterized by ventricular dilatation and impaired systolic function $(1,2)$. DCM is a leading cause of heart failure and arrhythmia. Due to its significant prevalence, high mortality and morbidity, including frequent hospitalizations, DCM is a major health concern for adults.

A progression from VMC to DCM has long been hypothesized, however, the true nature of this progression remains to be elucidated. However, a causal link between VMC and DCM is becoming more evident with significant developments in the molecular analyses of autopsy and endomyocardial biopsy specimens, and new techniques of viral gene amplification and modern immunology. The persistence of viral RNA in the myocardium 90 days after inoculation, confirmed by polymerase chain reaction (PCR), has provided new insights into the pathogenesis of DCM (3). Multiple mechanisms have been implicated (4), including direct viral injury and persistence, autoimmune phenomena, cytokine fluxes and T cell-mediated inflammatory responses.

Several studies have revealed that microRNAs (miRNAs) are essential for cardiac development and function (5-7). Furthermore, genetic studies have identified distinct roles for specific miRNAs during cardiogenesis, cardiac hypertrophy and electrical conduction $(8,9)$. This led the present study to hypothesize that miRNAs may be critical in the progression of VMC to DCM.

miRNAs have been reported to be important in diverse biological and pathological processes, including cell differentiation, proliferation, apoptosis, heart disease, neurological disorders and human cancer (10). Among the known miRNAs, 
the small regulatory RNA microRNA-21 (miRNA-21) is crucial in a number of biological functions and diseases, including development, cancer, cardiovascular diseases and inflammation. In previous studies, miRNA-21 has been revealed to be upregulated in various cardiovascular diseases. Cheng et al revealed that miRNA-21 affects PDCD4 in ischemic preconditioning (11). However, the role of miRNA-21 in the process of VMC and DCM is not completely understood.

Sprouty homolog (SPRY) is a novel protein family, which is primarily expressed in cardiac and skeletal muscles. The C-terminal SPRY domain and an N-terminal TRIM domain composes the structure of MG53 (12). SPRY was originally described as an antagonist of breathless FGF receptor signaling during tracheal branching in Drosophila (13). In total, four mammalian homologs (SPRY1-4) have been described and are widely expressed in embryonic and adult tissues (14). All SPRY proteins share a highly conserved, cysteine-rich C-terminal domain and a more variable N-terminal domain. They are subject to tight control at multiple levels, including differential localization, post-translational modification and the regulation of protein levels. SPRY participates in multiple physiological and pathological processes, including acute membrane repair, myogenesis in skeletal muscle and cardiac ischemic preconditioning $(12,15-17)$. Thum et al identified that SPRY1 was a direct target of miRNA-21 and mediated the effect of miRNA-21 in cardiac fibroblasts (18).

The present study assessed the expression of miRNA-21, SPRY and mitogen-activated protein kinase (MAPK) in a Sprague-Dawley mouse model of VMC and DCM of human left ventricular tissue, and investigated their potential roles in the pathology of the diseases. The expression of SPRY protein and its mRNA was also examined in cultured, miRNA-21 transfected myocardial cells.

\section{Materials and methods}

Mouse model of VMC. CVB3 viral stock derived from the infectious cDNA copy of the cardiotropic Nancy strain was used, as previously described (19). All experimental procedures were in compliance with the NIH Guide for the Care and Use of Laboratory Animals and approved by the Institutional Animal Care and Use Committee at Fudan University (Shanghai, China). In total, $30 \mathrm{BALB} / \mathrm{c}$ mice (male, 4-6 weeks old, 16-20 g) obtained from Fudan University were infected intraperitoneally with $5 \times 10^{4}$ plaque-forming units of purified CVB3, as described previously (20). In addition, 10 mice were infected intraperitoneally with Dulbecco's modified Eagle's medium (DMEM; Gibco-BRL, Carlsbad, CA, USA) as the control. Following 20 days, the mice were sacrificed by an intraperitoneal injection of pentobarbital $(40 \mathrm{mg} / \mathrm{kg})$ and the hearts were collected for analysis. Histology was performed on transverse tissue sections covering the right and left ventricle, obtained below the level of the valves, fixed in $4 \%$ phosphate-buffered paraformaldehyde ( $\mathrm{pH}$ 7.2) and embedded in paraffin. The remainders of the left and right ventricles were snap-frozen in liquid nitrogen and used for proteomic analysis.

Tissue acquisition. Human left ventricular tissue was collected from the Department of Forensic Medicine, Shanghai Medical College, Fudan University. Written, informed consent was obtained in advance from relatives. Samples were collected in the mortuary where cadavers were preserved at $4{ }^{\circ} \mathrm{C}$ following transport from the scene of death (criminal cases or traffic accidents). None of the cases had records of emergency treatment.

Subjects with DCM $(n=6)$ and non-VMC/DCM control subjects $(n=8)$ were collected. The diagnosis of DCM was made according to criteria provided by the World Health Organization/International Society and Federation of Cardiology (1), the Guidelines for the Study of Familial Dilated Cardiomyopathies (21) and the more recent Guidelines of the National Heart, Lung, and Blood Institute Workshop on the Prevalence and the Etiology of Idiopathic Dilated Cardiomyopathy (22) designed to improve the sensitivity and specificity of the old classification criteria. Patient demographic and clinical details are shown in Table I.

Histological analysis. The samples were embedded in phosphate-buffered paraformaldehyde and cut into $4-\mu \mathrm{m}$ thick sections. These were mounted on glass slides and stained with hematoxylin and eosin for histological examination.

RNA isolation. Total RNA was extracted with TRIzol, according to the manufacturer's instructions (Invitrogen Life Technologies, Carlsbad, CA, USA). Any remaining DNA was removed with the DNA-free kit (Ambion, Austin, TX, USA) and RNA was re-purified with an RNeasy kit, according to the manufacturer's instructions (Qiagen, Valencia, CA, USA).

Detection of miRNA-21 and SPRY mRNA by quantitative PCR $(q P C R)$. For quantification of the SPRY transcript, conventional qPCR was performed. The total RNA samples were extracted from the left ventricles or from cultured myocardial cells, as described below. TaqMan quantitative assay was performed with GAPDH expression as an internal control.

Total RNA was used to detect mature miRNA-21 expression using Hairpin-it miRNAs real-time PCR kit (Shanghai GenePharma Co., Ltd, Shanghai, China). In brief, the real-time miRNA assay consisted of two steps: stem-loop reverse transcription (RT) reaction and qPCR detection. Stem-loop RT primers bind to the $3^{\prime}$ end of miRNA molecules and are transcribed with reverse transcriptase. The RT product is quantified using qPCR, using an miRNA-specific forward primer, reverse primer and a carboxyfluorescein dye-labeled reporter probe. To normalize RNA content, the U6 snRNA was used as the internal control. The relative miRNA-21 expression levels in each group were calculated by the mathematical $\Delta \Delta$ method (23). The reactions were run three times for each group. The specific primers used were as follows: miR-21, forward 5'-TGGAATGTAAGGAAGTGTGTGGAC-3' and reverse 5'-CCAGTCTCAGGGTCCGAGGTATTC-3' (296 bp); U6, forward 5'-ATGACGTCTGCCTTGGAGAAC-3' and reverse 5'-TCAGTGTGCTACGGAGTTCAG-3' (291 bp); GAPDH, forward 5'-CTGACATGCCGCCTGGAGA-3' and reverse 5'-ATGTAGGCCATGAGGTCCAC-3' (260 bp); SPRY1 (NM_011896), forward 5'-ACCCTTCCTGTGTTTTCAT-3' and reverse 5'-AGTCACCTTGCTTTTCTTG-3' (mouse); SPRY1 (NM_005841),forward5'-GGACCTGACACAGCACAAGTT-3' and reverse 5'-AGATGCCCTTGACTAAGCACA-3' (170 bp; human). 
Table I. Patient demographic and clinical details.

\begin{tabular}{|c|c|c|c|c|c|c|}
\hline Patient no. & Gender & $\begin{array}{l}\text { Age, } \\
\text { years }\end{array}$ & $\begin{array}{l}\text { Sodium, } \\
\mathrm{mmol} / \mathrm{l}\end{array}$ & $\begin{array}{l}\text { Creatinine, } \\
\qquad \mu \mathrm{mol} / 1\end{array}$ & $\begin{array}{l}\text { GLU, } \\
\mathrm{mmol} / 1\end{array}$ & $\begin{array}{c}\text { Triglycerides, } \\
\mathrm{mmol} / \mathrm{l}\end{array}$ \\
\hline \multicolumn{7}{|l|}{ DCM } \\
\hline 1 & $\mathrm{M}$ & 42 & 142 & 67 & 4.4 & 1.8 \\
\hline 2 & $\mathrm{M}$ & 36 & 141 & 89 & 5.0 & 1.3 \\
\hline 3 & $\mathrm{~F}$ & 60 & 135 & 102 & 4.1 & 1.7 \\
\hline 4 & $\mathrm{M}$ & 57 & 141 & 57 & 6.0 & 1.2 \\
\hline 5 & $\mathrm{M}$ & 24 & 136 & 74 & 4.8 & 0.8 \\
\hline 6 & $\mathrm{~F}$ & 50 & 140 & 42 & 5.8 & 1.4 \\
\hline \multicolumn{7}{|l|}{ Control } \\
\hline $7^{\mathrm{a}}$ & $\mathrm{M}$ & 52 & 142 & 88 & 5.5 & 1.6 \\
\hline $8^{b}$ & $\mathrm{~F}$ & 43 & 146 & 97 & 4.5 & 0.9 \\
\hline $9^{a}$ & $\mathrm{~F}$ & 23 & 135 & 56 & 4.1 & 1.1 \\
\hline $10^{c}$ & M & 19 & 147 & 60 & 4.2 & 0.7 \\
\hline $11^{\mathrm{a}}$ & M & 32 & 144 & 70 & 3.4 & 0.9 \\
\hline $12^{\mathrm{a}}$ & $\mathrm{M}$ & 58 & 132 & 91 & 5.8 & 1.3 \\
\hline $13^{\mathrm{a}}$ & $\mathrm{M}$ & 38 & 131 & 66 & 4.1 & 1.1 \\
\hline $14^{\mathrm{d}}$ & $\mathrm{F}$ & 62 & 149 & 92 & 6.2 & 1.5 \\
\hline
\end{tabular}

${ }^{\mathrm{a}}$ Healthy heart, ${ }^{\mathrm{b}}$ coronary artery disease, ${ }^{\mathrm{c}}$ rheumatic heart disease and ${ }^{\mathrm{d} h y p e r t e n s i v e ~ h e a r t ~ d i s e a s e . ~ D C M, ~ d i l a t e d ~ c a r d i o m y o p a t h y ; ~ G L U, ~ g l u c o s e ~}$

The predicted target genes and their miRNA binding site seed regions were investigated using TargetScan (release 5.1, http://www.targetscan.org/). The sequences of the predicted mature miRNAs were confirmed using miRBase (release 16.0, September 2010; http://microrna.sanger.ac.uk/).

Western blotting for SPRY and MAPK expression. The protein extract $(15 \mathrm{~g})$ prepared from tissue samples or cultured heart cells was examined by western blot analysis. Equal amounts of protein were subjected to SDS-PAGE. A standard western blot analysis was conducted using a mouse monoclonal SPRY antibody (Millipore, Billerica, MA, USA). A mouse polyclonal GADPH antibody (1:5000 dilution; Kangcheng Inc., Shanghai, China) was used as the loading control.

Cell isolation from neonatal rat heart and primary cell culture. Neonatal rat ventricular cardiomyocytes were isolated and cultured as described previously (24). Briefly, 1-2 day old Sprague-Dawley rats obtained from Fudan University were placed in ice-cold $1 \mathrm{X}$ phosphate-buffered saline solution. Following repeated rinsing, rats were decapitated, the hearts were aseptically removed, dissected, the atria were cut off and the ventricles were minced with scissors. The minced tissue and ventricular cells were dispersed by digestion with collagenase type IV $(0.45 \mathrm{mg} / \mathrm{ml})$ and $0.1 \%$ trypsin. Cardiomyocytes $\left(0.33 \times 10^{6}\right.$ cells $\left./ \mathrm{ml}\right)$ were cultured in cardiac myocyte culture medium containing DMEM/F-12 (Gibco-BRL) supplemented with $4 \mathrm{mg} / \mathrm{ml}$ transferrin, $0.7 \mathrm{ng} / \mathrm{ml}$ sodium selenite, $2 \mathrm{~g} / \mathrm{l}$ bovine serum albumin, $3 \mathrm{mmol} / 1$ pyruvic acid, $15 \mathrm{mmol} / 1$ HEPES, $100 \mathrm{mmol} / 1$ ascorbic acid, $100 \mathrm{mg} / \mathrm{ml}$ ampicillin, $5 \mathrm{mg} / \mathrm{ml} \mathrm{linoleic} \mathrm{acid,} 1 \%$ penicillin, $1 \%$ streptomycin and $100 \mathrm{mmol} / 1$ 5-bromo-2'-deoxyuridine, and seeded into six-well plates. Hypoxia was achieved by placing the cells in a hypoxia chamber filled with $5 \% \mathrm{CO}_{2}$ and $95 \% \mathrm{~N}_{2}$ at $37^{\circ} \mathrm{C}$ for $4 \mathrm{~h}$. Following hypoxia exposure, the cells were reoxygenated with $5 \% \mathrm{CO}_{2}$ and $95 \% \mathrm{O}_{2}$ for $3 \mathrm{~h}$ in DMEM containing $5 \%$ serum and normal glucose.

Synthesis of miRNAs and sequences of the miRNA inhibitor. Mouse miRNA-21 and its mutant RNA oligos were synthesized by Integrated DNA Technologies, Inc. (Coralville, IA, USA). The antisense oligonucleotide for miRNA-21, 2'OMe-miRNA-21 or the miRNA inhibitor, was modified at each nucleotide by an O-methyl moiety at the 2'ribose position: 5'-UmCmAmAmCmAmUmCm AmGmUmCmUmGmAmUmAmAmGmCmUmA-3'. In addition, a control modified antisense oligonucleotide was used for enhanced green fluorescence protein (EGFP) mRNA (2'OMe-EGFP): 5'mAmAmGmGmCmAmAm-GmCmUmG-

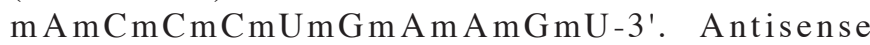
inhibitor oligonucleotide (AMO)-21 and mis-AMO-21 contained 2'-O-methyl modifications at every base and a 3' C3-containing amino linker.

Transfection of miRNAs. miRNA-21 and its mutant constructs were synthesized by Integrated DNA Technologies, Inc. The sequences of anti-miRNA-21 antisense inhibitor oligonucleotides (AMOs) used in our studies are the exact antisense copies of mature miRNA sequences, have 2'-O-methyl modifications at every base and a $3^{\prime} \mathrm{C} 3$ containing amino linker $(25,26)$. Oligo transfection was performed according to the manufacturer's instructions (27). The cells were transfected using transfection reagent, miR-21 mimics, negative control miRNA mimics or stabilized miRNAs (Ago-miR-21 and Ago-miR-NC) purchased from Guangzhou RiboBio Co., Ltd. (Guangzhou, Guangdong, China). Transfection complexes 
were prepared according to the manufacturer's instructions, and 2'OMe-miRNA-21 or control oligo 2'OMe-EGFP was added to a final oligonucleotide concentration of 30,50 and $100 \mathrm{nmol} / \mathrm{l}$. The transfection efficiency was low at 30 or $50 \mathrm{nmol} / \mathrm{l}$. However, at a concentration of $100 \mathrm{nmol} / \mathrm{l}$, the transfection efficiency was at least $80 \%$. Therefore, this concentration was selected for our experiments. The transfection medium was replaced with the normal culture medium following $6 \mathrm{~h}$ of incubation.

Statistical analysis. Statistical analysis was performed using SPSS 10.5 (SPSS, Inc., Chicago, IL, USA). Data are presented as the mean \pm standard deviation. Differences between groups were assessed using one-way analysis of variance. $\mathrm{P}<0.05$ was considered to indicate a statistically significant difference. All experiments were performed at least three times.

\section{Results}

Establishment of VMC in mice. Following virus inoculation, signs of VMC were apparent in the experimental groups following 3 days, including coat ruffling, weakness and irritability. On day 4, a few scattered small foci of myocyte necrosis were noted on histological analysis. On day 10, myocardial necrosis and cell infiltration were extensive and necrotic areas appeared more prominent. There were numerous lymphocytes and macrophages in and around the necrotic foci. On day 20, inflammatory cells and necrotic areas decreased and necrotic myocardium gradually became fibrotic and calcified (Fig. 1B). There were no necrotic lesions or signs of cell infiltration in the hearts of uninfected control mice (Fig. 1A). On day 20 disease progressed from acute to chronic VMC and was the time point at which inflammation peaked. Therefore, day 20 post-infection was selected as the time point for further evaluation.

miRNA-21 expression in VMC and DCM. The mechanism of action of miRNAs involves the incorporation of the single-stranded miRNA into the RNA-induced silencing complex and its subsequent binding to the 3 ' untranslated region (3'-UTR) of the target mRNA through exact complementarity with the 7-8 nt of the 5 ' end, and partial complementarity with the rest of the sequence (28-30). In this way, miRNAs achieve translational inhibition. In order to examine the possible involvement of miRNAs in the regulation of SPRY1 expression, a bioinformatics based approach was used to predict the putative targets, employing TargetScan hosted by the Wellcome Trust Sanger Institute. Identical potential binding sites were identified for mouse miRNA-21 and SPRY1. It was revealed that the expression level of miRNA-21 increased by $300 \%$ in cardiac ventricle tissues of VMC mice (relative expression: $13 \pm 2.5$ vs. $4 \pm 1.6$; $\mathrm{P}<0.05$; Fig. $2 \mathrm{~A}$ ) and by $800 \%$ in cardiac ventricle tissues of DCM (relative expression: $16 \pm 0.315$ vs. $2 \pm 0.273, \mathrm{P}<0.05$; Fig. 2B).

Expression of the SPRY and MAPK proteins in VMC mice. Western blotting was performed for SPRY and MAPK protein in the mouse model of VMC. The expression of the SPRY protein decreased significantly 20 days after virus inoculation $(\mathrm{P}<0.05$; Fig. 3A). However, the expression of the MAPK protein increased significantly at the same time point $(\mathrm{P}<0.05$; Fig. $3 \mathrm{~B})$.
A

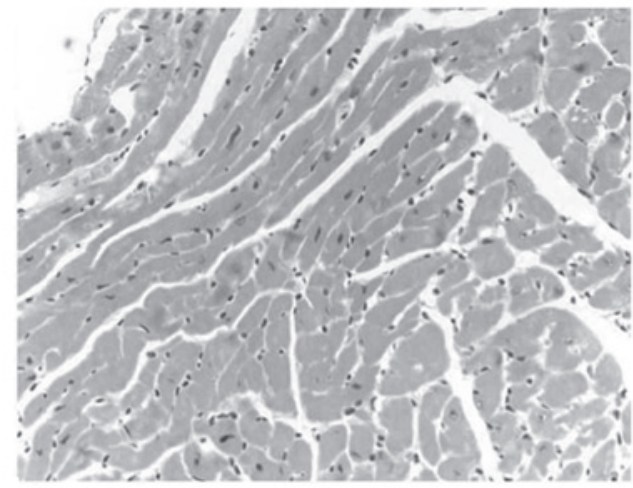

B

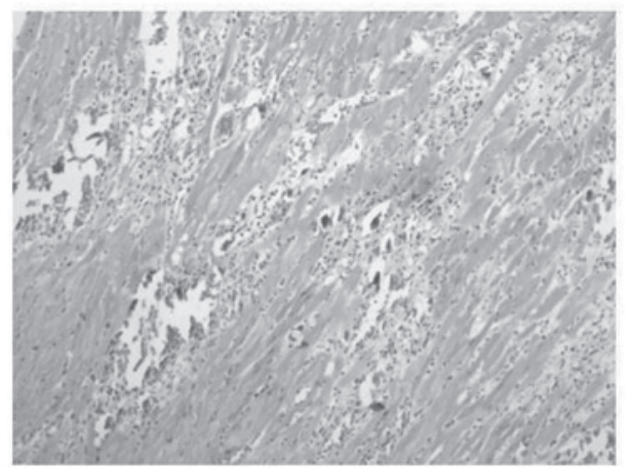

Figure 1. (A) H\&E stain of the paraffin-embedded left ventricle from a control group heart (original magnification, x100). (B) H\&E stain of the paraffin-embedded left ventricle from a VMC heart. Numerous lymphocytes and macrophages infiltrated the cardiac tissue on day 20 (original magnification, x100). H\&E, hematoxylin and eosin; VMC, viral myocarditis.

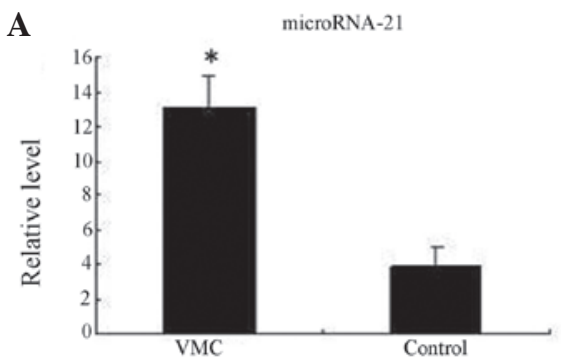

B

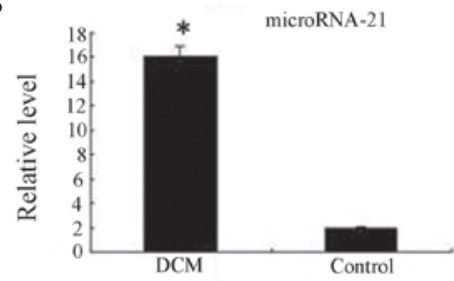

Figure 2. (A) miRNA-21 expression was greatly increased in VMC mice $($ ( $\mathrm{P}<0.05)$. Results were normalized to the expression of U6. (B) miRNA-21 expression was greatly increased in DCM human left ventricular tissue $\left({ }^{*} \mathrm{P}<0.05\right)$. Results were normalized to the expression of U6. miRNA-21, microRNA-21; VMC, viral myocarditis; DCM, dilated cardiomyopathy.

Notably, the changes in SPRY1 expression at the mRNA level did not reflect the protein expression level. qPCR demonstrated a significantly smaller decrease in the level of the SPRY1 
A
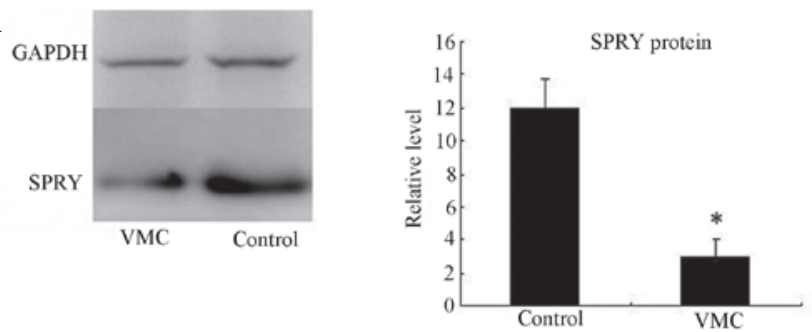

B
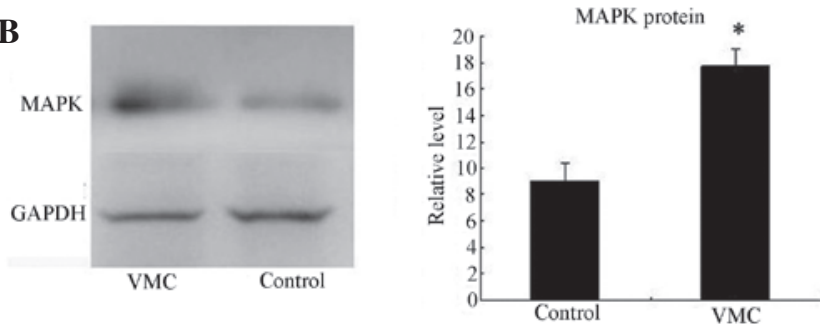

C

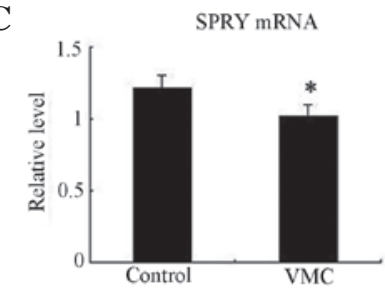

Figure 3. (A) Comparisons of SPRY protein levels between membrane samples isolated from mice with healthy control hearts and from mice with VMC, determined by western blot analysis. The results are expressed as the mean \pm SE from 10 controls and $30 \mathrm{VMCs} ;{ }^{*} \mathrm{P}<0.05$ vs. control; unpaired Student's t-test. (B) Comparisons of MAPK protein levels between healthy control hearts and mice with VMC, determined by western blot analysis. The results are expressed as the mean \pm SEM from 10 controls and $30 \mathrm{VMCs}$; ${ }^{*} \mathrm{P}<0.05$ vs. control; unpaired Student's t-test. (C) There was a slightly decreased expression of SPRY1 at the mRNA levels (not significant, P $>0.05$ vs. control; unpaired Student's t-test). The results were normalized to the expression of GAPDH. SPRY, sprouty; VMC, viral myocarditis; MAPK, mitogen-activated protein kinase.

transcript than that of its protein (Fig. 3C). The SPRY protein expression diminished almost four-fold $(\mathrm{P}<0.05)$; however, only a 20\% ( $>0.05)$ decrease in the mRNA levels was observed.

These results suggest a post-transcriptional mechanism regulating the expression of the SPRY1 gene in VMC mice.

Expression of the SPRY and MAPK proteins in DCM. Western blotting was also performed for SPRY and MAPK proteins in human left ventricular tissue with DCM. It was revealed that the expression of the SPRY protein in DCM was significantly lower than that in the control tissue $(\mathrm{P}<0.05$; Fig. 4A). MAPK protein levels were significantly higher in DCM than in controls $(\mathrm{P}<0.05$; Fig. 4B). Similar results were obtained with VMC tissue.

Similarly, the decrease in the expression of SPRY1 mRNA levels was significantly smaller than the decrease in protein levels (Fig. 4C).

Post-transcriptional repression of SPRY1 by miRNA-21. The results above demonstrate that the expression of SPRY, MAPK protein and miRNA-21 change in VMC and DCM. The ability of miRNA-21 to repress the expression of SPRY1
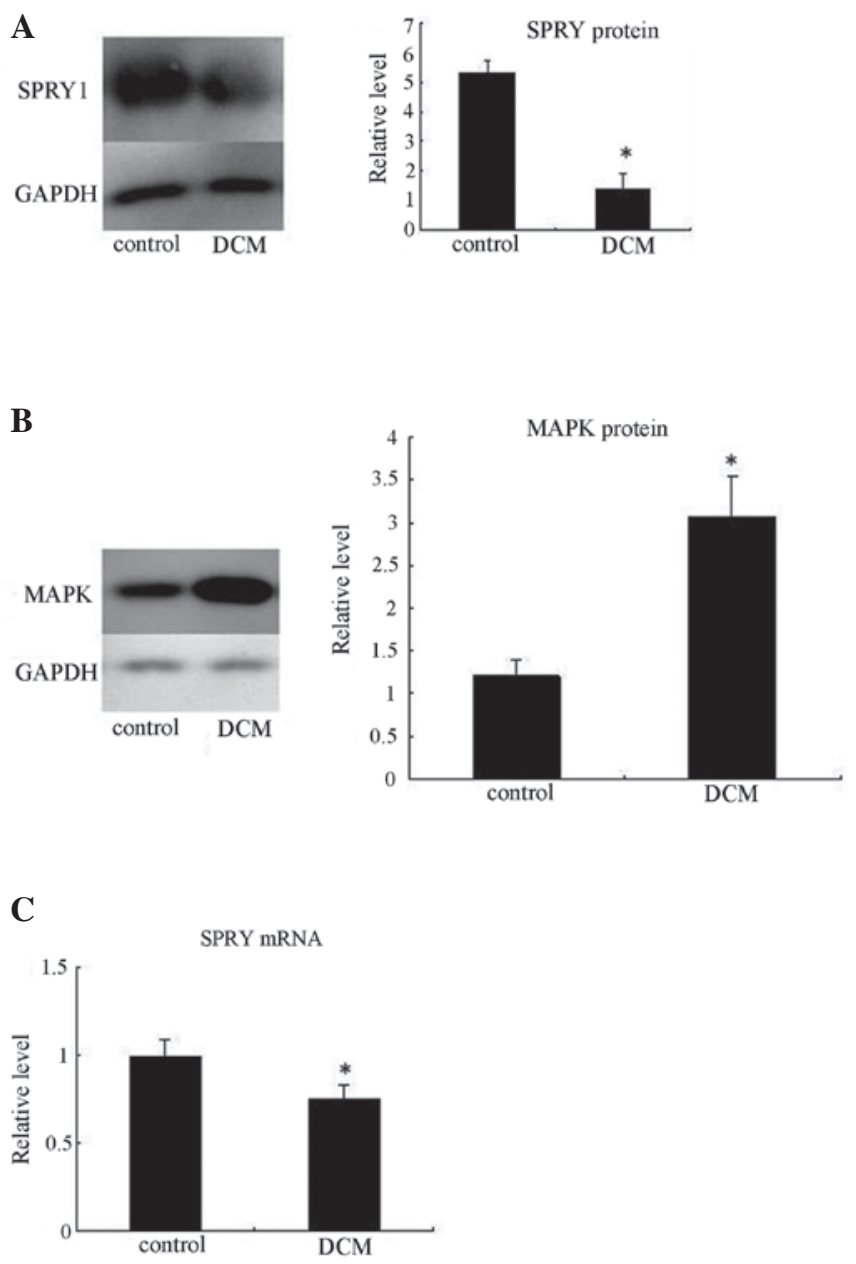

Figure 4. (A) Comparisons of SPRY protein levels between human left ventricular tissue with DCM and control hearts, determined by western blot analysis. The results are expressed as the mean \pm SE from six controls and six DCMs; ${ }^{*} \mathrm{P}<0.05$ vs. control; unpaired Student's t-test. (B) Comparisons of MAPK protein levels between human left ventricular tissue with DCM and control hearts, determined by western blot analysis. The results are expressed as the mean \pm SEM from six control and six DCMs; ${ }^{*} \mathrm{P}<0.05$ vs. control; unpaired Student's t-test. (C) There was a slightly decreased expression of SPRY1 at the mRNA level (not significant, $\mathrm{P}>0.05$ vs. control; unpaired Student's t-test). The results were normalized to the expression of GAPDH. SPRY, sprouty; DCM, dilated cardiomyopathy; MAPK, mitogen-activated protein kinase.

in cardiac myocytes was also evaluated. As shown in Fig. 5B, the transfection of miRNA-21 into cultured cardiac myocytes substantially reduced SPRY protein levels. The transfection with mis-miRNA-21 (negative control) did not affect SPRY expression. Furthermore, when the anti-miRNA-21 AMO-1 was co-transfected with miRNA-21, it almost eradicated the effect of miRNA-21, thus, verifying the specificity of the effects of miRNA-21. Co-transfection of mis-AMO-21 (negative control) with miRNA-21 reduced the levels of the SPRY protein (Fig. 6A); no cancellation of the miRNA-21 effect was observed. By contrast, miRNA-21 exerted virtually no effect on SPRY1 mRNA levels (Fig. 6B), indicating that it does not affect SPRY1 mRNA stability.

\section{Discussion}

The major new findings of the present study were: i) miRNA-21 was upregulated in the mouse model of VMC and patients 

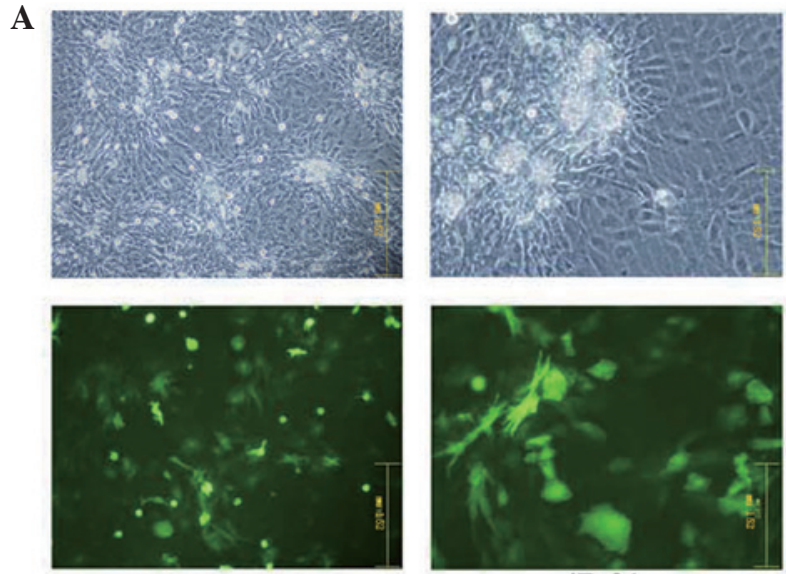

miR-control

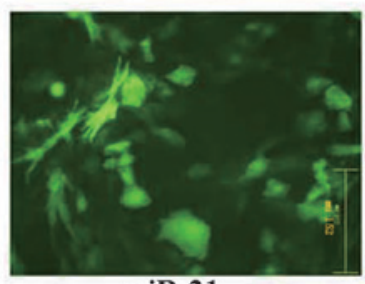

miR-21

B
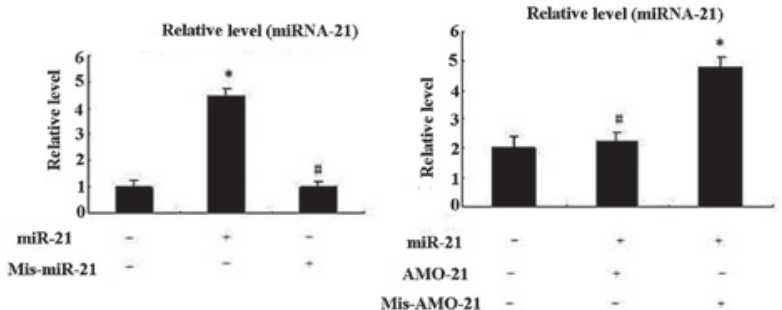

Figure 5. Post-transcriptional repression of SPRY1 by miRNA-21. (A) miRNA-control (negative control), miRNA-21 and AMO-21 were transduced into neonatal rat cardiac myocytes. The transduction efficiency, which is shown in the image, was always $>80 \%$. (B) Levels of miRNA- 21 in cultured cardiac myocytes that were transfected with miRNA-21 alone or with miRNA-21 and mis-miRNA-21, AMO-21 or mis-AMO-21. Data are presented as the mean $\pm \mathrm{SEM}\left(\mathrm{n}=5\right.$ independent samples for each group); ${ }^{*} \mathrm{P}<0.05$ vs. control, ${ }^{\prime} \mathrm{P}>0.05$ vs. control; unpaired Student's t-test. miRNA-21, microRNA-21; AMO, antisense inhibitor oligonucleotides; SPRY, sprouty.

with DC; ii) SPRY protein levels were decreased, but MAPK protein levels were increased in VMC and DCM, and the changes in SPRY1 expression at the mRNA level did not reflect the protein expression levels; and iii) the target of miRNA-21 was SPRY1, and miRNA-21, through post-transcriptional mechanisms, inhibits the SPRY protein and enhances the MAPK signaling pathway.

miRNA biogenesis begins with the transcription of primary miRNAs (pri-miRNAs) by RNA polymerase II as long transcripts. In the nucleus, the pri-miRNAs are cleaved by RNase III endonuclease Drosha and the dsRNA-binding protein DGCR8 to produce 70 -nt long intermediate precursor-miRNAs (pre-miRNAs) with a characteristic stem-loop structure (31). Pre-miRNAs are then exported to the cytoplasm by exportin- 5 and further cleaved by Dicer, another RNase III endonuclease (32). The resulting 20-23 nt mature miRNA duplex is released by Dicer and a single stem-arm (the guiding strand) is incorporated into the RNA-induced silencing complex, which recognizes specific targets by binding to their 3' UTRs and induces post-transcriptional gene silencing (33). In certain cases, miRNAs are also known to bind to the 3'UTRs and the coding sequences, or the open reading frames, of their target genes.

miRNAs are involved in the regulation of diverse cellular processes, including proliferation, differentiation, cellular
A
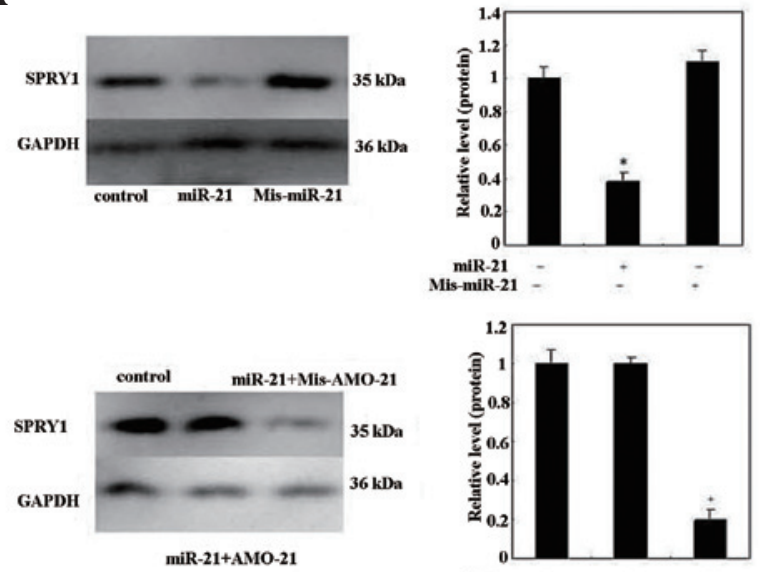

miR-21

AMO-21

Mls-AMO-21

\section{B}

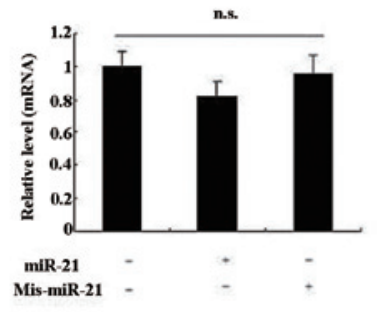

Figure 6. (A) SPRY protein levels were determined by western blotting on protein extracts from cultured neonatal rat ventricular myocytes (32). Cells were transfected with miRNA-21 alone or with miRNA-21 and mis-miRNA-21, AMO-21 or mis-AMO-21. AMO-21 reversed the repressive effects of miRNA-21 on SPRY, however, mis-AMO-21 did not. Mean \pm SEM from five batches of cells. ${ }^{*} \mathrm{P}<0.05$ versus control, ${ }^{+} \mathrm{P}<0.05$ versus miRNA-21 alone; unpaired Student's t-test. (B) Quantitative polymerase chain reaction analysis demonstrated no significant changes in mRNA levels of SPRY1 in miRNA-21 overexpressing cardiac myocytes. Values are the means \pm SEM of 5-6 independent experiments. miRNA-21, microRNA-21; AMO, antisense inhibitor oligonucleotides; SPRY, sprouty; n.s. not significant.

migration and apoptosis. Under cell stress conditions, the deregulation of miRNAs is often observed and may result in the development of disease. Several studies revealed that miRNAs are essential for vascular signaling, cardiac development and function. Furthermore, genetic studies have identified distinct roles for specific miRNAs during cardiogenesis, cardiac hypertrophy and electrical conduction.

Our previous study demonstrated the role of protein degradation in the pathogenesis of VMC and its progression to DCM $(34,35)$. These abnormalities trigger a cascade of events that may ultimately contribute to the transition of VMC to DCM.

A progression from VMC to DCM has long been hypothesized, however, definitive evidence is lacking. However, a causal link between VMC and DCM is becoming more evident with marked developments in the molecular analyses of autopsy and endomyocardial biopsy specimens, new techniques of viral gene amplification and modern immunology. At present, the mechanisms underlying the transition of VMC to DCM include direct viral injury and persistence, autoimmune phenomena, cytokine fluxes and T-cell mediated inflammatory responses (36). However, whether miRNAs are also critical in the pathogenesis of VMC leading to DCM remains to be eluci- 
dated. Taking into account our own previous results, our data suggests that miRNAs are important during the progression from VMC to DCM, involving an inappropriate repression or derepression of crucial protein targets. This is a novel pathophysiological mechanism in the two diseases.

SPRY belongs to the sprouty family and is a negative feedback regulator of the MAPK signaling pathway. The inhibition of MAPK signaling through the CDK/MAPK/GSK3/CLK kinase group is one of the pathways by which the SPRY signal is transduced to the nucleus and regulates the expression of genes. SPRY regulates the expression of the collagen gene and regulates cell growth, differentiation, transformation, proliferation, cell survival and apoptosis (37).

On the basis of these data, the present study proposes that the abnormal expression of miRNA-21 in myocardial cells inhibits SPRY protein expression, resulting in the augmentation of MAPK expression. The 3' UTR of SPRY1 mRNA contains a predicted miRNA-binding site with miRNA-21. SPRY1 is a direct target of miRNA-21. The effects of miRNA-21 upregulation during cardiac disease had no detectable effect on SPRY1 mRNA level, however, resulted in the strong repression of SPRY protein expression. SPRY specifically inhibits MAPK expression, therefore, the increased expression of MAPK leads to myocardial fibrosis and cardiac remodelling. Myocardial fibrosis is the most important cause of heart disease arrhythmia, cardiac dysfunction, other complications and even sudden cardiac death (38). These results present a novel view that miRNA-21 levels are increased in VMC and DCM, and that it may be important in cardiac fibrosis in these diseases. It is also important in the progression of VMC to DCM. To the best of our knowledge, this is the first study examining the role of miRNAs in the pathogenic progression of VMC to DCM.

In summary, the present study demonstrated that miRNA-21 levels are increased in VMC and DCM. Our data suggests that miRNA-21, through the inhibition of the SPRY protein, affects the MAPK signaling pathway. The regulation of miRNAs may contribute to the pathogenic progression of VMC to DCM. These findings suggest a new therapeutic entry point for cardiac disease and illustrate the broad therapeutic potential of miRNA modulation.

\section{Acknowledgements}

This study was supported by the National Science Foundation of China (nos. 81172897 and 81302617) and the Natural Science Foundation of Nantong (no. BK2011053).

\section{References}

1. Richardson P, McKenna W, Bristow M, et al: Report of the 1995 World Health Organization/International Society and Federation of Cardiolgy Task Force on the definition and classification of cardiomyopathies. Circulation 93: 841-842, 1996.

2. Feldman AM and McNamara D: Myocarditis. N Engl Med 343: 1388-1398, 2000.

3. Kawai C: From myocarditis to cardiomyopathy: mechanisms of inflammation and cell death. Circulation 99: 1091-1100, 1999.

4. Miyamoto SD, DeBiasi RL and Long CS: Novel therapeutic targets in viral myocarditis. Future Virol 3: 373-381, 2008.

5. Zhao Y, Samal E and Srivastava D: Serum response factor regulates a muscle-specific microRNA that targets Hand2 during cardiogenesis. Nature 436: 214-222, 2005.
6. Lin Z, Murtaza I, Wang K, et al: miR-23a functions downstream of NFATc3 to regulate cardiac hypertrophy. Proc Natl Acad Sci USA 106: 12103-12108, 2009.

7. Shan ZX, Lin QX, Fu YH, et al: Upregulated expression of $\mathrm{miR}-1 / \mathrm{miR}-206$ in a rat model of myocardial infarction. Biochem Biophys Res Commun 381: 597-601, 2009.

8. Goren Y, Kushnir M, Zafrir B, Tabak S, Lewis BS and Amir O: Serum levels of microRNAs in patients with heart failure. Eur J Heart Fail 14: 147-154, 2012.

9. Seto AG and van Rooij E: Circulating microRNAs to identify human heart failure. Eur J Heart Fail 14: 118-119, 2012.

10. Karp X and Ambros V: Developmental biology: encountering microRNAs in cell fate signaling. Science 310: 1288-1289, 2005.

11. Cheng Y, Zhu P, Yang J, Liu X, Dong S, Wang X, Chun B, Zhuang $J$ and Zhang $C$ : Ischaemic preconditioning-regulated miR-21 protects heart against ischaemia/reperfusion injury via anti-apoptosis through its target PDCD4. Cardiovasc Res 87: 431-439, 2010.

12. Cai C, Masumiya H, Weisleder N, et al: MG53 nucleates assembly of cell membrane repair machinery. Nat Cell Biol 11: 56-64, 2009.

13. Hacohen N, Kramer S, Sutherland D, Hiromi Y and Krasnow MA: Sprouty encodes a novel antagonist of FGF signaling that patterns apical branching of the Drosophila airways. Cell 92: 253-263, 1998.

14. Minowada G, Jarvis LA, Chi CL, Neubüser A, Sun X, Hacohen N, Krasnow MA and Martin GR: Vertebrate Sprouty genes are induced by FGF signaling and can cause chondrodysplasia when overexpressed. Development 126: 4465-4475, 1999.

15. Cai C, Masumiya H, Weisleder N, Pan Z, Nishi M, Komazaki S, Takeshima $\mathrm{H}$ and Ma J: MG53 regulates membrane budding and exocytosis in muscle cells. J Biol Chem 284: 3314-3322, 2009.

16. Cai C, Weisleder N, Ko JK, Komazaki S, Sunada Y, Nishi M, Takeshima $\mathrm{H}$ and Ma J: Membrane repair defects in muscular dystrophy are linked to altered interaction between MG53, caveolin-3 and dysferlin. J Biol Chem 284: 15894-15902, 2009.

17. Cao C, Zhang Y, Weisleder N, et al: MG53 Constitutes a primary determinant of cardiac ischemic preconditioning. Circulation 121: 2565-2574, 2010.

18. Thum T, Gross C, Fiedler J, et al: MicroRNA-21 contributes to myocardial disease by stimulating MAP kinase signalling in fibroblasts. Nature 456: 980-984, 2008.

19. Klingel K, Hohenadl C, Canu A, Albrecht M, Seemann M, Mall G and Kandolf R: Ongoing enterovirus-induced myocarditis is associated with persistent heart muscle infection: quantitative analysis of virus replication, tissue damage, and inflammation. Proc Natl Acad Sci USA 89: 314-318, 1992.

20. Kandolf R, Selinka $\mathrm{H}$ and Klingel K: Pathogenesis of Coxsackievirus B infections. In: Molecular Biology of Picornaviruses. Semler BL and Wimmer E (eds). American Society of Microbiology, Washington, DC, pp 405-413, 2002.

21. Mestroni L, Maisch B, McKenna WJ, Schwartz K, Charron P, Rocco C, Tesson F, Richter A, Wilke A and Komajda M: Guidelines for the study of familial dilated cardiomyopathies. Collaborative Research Group of the European Human and Capital Mobility Project on Familial Dilated Cardiomyopathy. Eur Heart J 20: 93-102, 1999.

22. McMurray, JJV, Adamopoulos S, Anker SD, et al: ESC guidelines for the diagnosis and treatment of acute and chronic heart failure 2012: The Task Force for the Diagnosis and Treatment of Acute and Chronic Heart Failure 2012 of the European Society of Cardiology. Developed in collaboration with the Heart Failure Association (HFA) of the ESC. Eur J Heart Fail 14: 803-869, 2012.

23. Pfaffl MW: A new mathematical model for relative quantification in real-time RT-PCR. Nucleic Acids Res 29: e45, 2001.

24. Pang L, Koren G, Wang Z and Nattel S: Tissue-specific expression of two human $\mathrm{Ca}(\mathrm{v}) 1.2$ isoforms under the control of distinct $5^{\prime}$ flanking regulatory elements. FEBS Lett 546: 349-354, 2003.

25. Luo X, Xiao J, Lin H, Li B, Lu Y, Yang B and Wang Z: Transcriptional activation by stimulating protein 1 and post-transcriptional repression by muscle-specific microRNAs of IKs-encoding genes and potential implications in regional heterogeneity of their expressions. J Cell Physiol 212: 358-367, 2007.

26. Caplen NJ, Parrish S, Imani F, Fire A and Morgan RA: Specific inhibition of gene expression by small double-stranded RNAs in invertebrate and vertebrate systems. Proc Natl Acad Sci USA 98: 9742-9747, 2001.

27. Lewis BP, Shih IH, Jones-Rhoades MW, Bartel DP and Burge CB: Prediction of mammalian microRNA targets. Cell 115: 787-798, 2003. 
28. Brennecke J, Stark A, Russell RB and Cohen SM: Principles of microRNA-target recognition. PLoS Biol 3: e85, 2005.

29. Cannell IG, Kong YW and Bushell M: How do microRNAs regulate gene expression? Biochem Soc Trans 36: 1224-1231, 2008.

30. Griffiths-Jones S: The microRNA registry. Nucleic Acids Res 32: D109-D111, 2004.

31. Lee Y, Ahn C, Han J, Choi H, Kim J, Yim J, Lee J, Provost P, Rådmark O, Kim S and Kim VN: The nuclear RNase III Drosha initiates microRNA processing. Nature 425: 415-419, 2003.

32. Lund E, Güttinger S, Calado A, Dahlberg JE and Kutay U: Nuclear export of microRNA precursors. Science 303: 95-98, 2004.

33. Khvorova A, Reynolds A and Jayasena SD: Functional siRNAs and miRNAs exhibit strand bias. Cell 115: 209-216, 2003

34. Xu HF, Li YH, Chen Y and Cheng LB: The expression of dystrophin in human viral myocarditis and dilated cardiomyopathy. Fa Yi Xue Za Zhi 22: 12-14, 2006 (In Chinese).
35. Xu HF; Chen JL; Da XP, Wu KR, Liu GQ, Zhao ZQ and Han XH: Expression of CAR in myocardial of viral myocarditis and dilated cardiomyopathy. Fa Yi Xue Za Zhi 26: 328-331, 2010 (In Chinese).

36. Kawai C: From myocarditis to cardiomyopathy: mechanisms of inflammation and cell death: learning from the past for the future. Circulation 99: 1091-1100, 1999.

37. Pearson G, Robinson F, Beers Gibson T, Xu BE, Karandikar M, Berman K and Cobb MH: Mitogen-activated protein (MAP) kinase pathways: regulation and physiological functions. Endocr Rev 22: 153-183, 2001

38. Heldin $\mathrm{CH}$, Miyazono $\mathrm{K}$ and ten Dijke P: TGF-beta signaling from cell membrane to nucleus through SMAD proteins. Nature 390: 465-471, 1997. 